\title{
Exhaled 15-HETE and thromboxin-B2 are associated with the therapeutic outcome in childhood asthma
}

\author{
Li-Chen Chen ${ }^{1}$, Hsu-Min Tseng ${ }^{2}$, Ming-Ling Kuo ${ }^{1}$, Chih-Yung Chiu ${ }^{3}$, Sui-Ling Liao ${ }^{3}$, \\ Kuan-Wen $\mathrm{Su}^{3}$, Ming-Han Tsai ${ }^{3}$, Man Chin Hua ${ }^{3}$, Shen-Hao Lai ${ }^{3}$, Tsung-Chieh $\mathrm{Yao}^{4}$, \\ Kuo-Wei Yeh ${ }^{4}$, Ai-Hsuan $\mathrm{Wu}^{1}$, Hsiu-Yueh $\mathrm{Yu}^{1}$, Jing-Long Huang ${ }^{1}$, and Shau-Ku Huang ${ }^{5}$ \\ ${ }^{1}$ New Taipei City Municipal Tucheng Hospital \\ ${ }^{2}$ Chang Gung University \\ ${ }^{3}$ Chang Gung Memorial Hospital Keelung Branch Library \\ ${ }^{4}$ Chang Gung Memorial Hospital Linkou Main Branch \\ ${ }^{5}$ National Health Research Institutes
}

April 1, 2021

\begin{abstract}
Background: Dysregulation of eicosanoids is associated with asthma and a composite of oxylipins, including exhaled LTB4, characterizes childhood asthma. While FeNO has been used as the standard for monitoring steroid responsiveness, the potential utility of eicosanoids in monitoring the therapeutic outcomes remains unclear. We aimed to examine the levels of major eicosanoids representing different metabolic pathways in exhaled breath condensates (EBCs) of children with asthma during exacerbation and after treatment. Methods: Levels of 6 exhaled eicosanoid species in asthmatic children and healthy subjects were evaluated using ELISA. Results: In addition to those previously reported, including LTB4, the levels of exhaled 15HETE, but not TXB2, showed significant difference between asthmatics $(\mathrm{N}=318)$ and healthy controls $(\mathrm{N}=97)$, particularly the severe group showed the lowest levels of exhaled 15-HETE. Receiver Operating Characteristic (ROC) analyses revealed similar distinguishing power for the levels of 15-HETE, FEV1 and FeNO, whilethe 15-HETE/LTB4 ratio was significantly lower in subjects with severe asthma $(\mathrm{p}<0.01)$. Analysis of asthmatics $(\mathrm{N}=75)$ during exacerbation and convalescence showed significant improvement in lung function (FEV1; $<<0.001$ ), but not FeNO, concomitant with significantly increased levels of 15-HETE $(\mathrm{p}<0.001)$ and reduced levels of TXB2 $(\mathrm{p}<0.05)$ after therapy, particularly for those who at the top $30 \%$ level during exacerbation. Further, decreased LTB4 and LXA4 at convalescence were noted only in those at the top 30 percentile during exacerbation. Conclusion: The exhaled 15-HETE was found to discriminate childhood asthma while decreased levels of exhaled TXB2 and increased levels of 15-HETE were prominent after treatment.
\end{abstract}

\section{Introduction}

Asthma is a chronic inflammatory disorder of the airways and is characterized by airway hyperresponsiveness and reversible airflow obstruction that fluctuates over time. It is also recognized as a heterogeneous disease with varying severity, responsiveness to therapy, and long-term outcome ${ }^{1}$. Eicosanoids are a family of bioactive lipid mediators that regulate a wide variety of inflammatory processes ${ }^{2}$. Eicosanoid species are generated from polyunsaturated fatty acids (PUFAs), such as arachidonic acid (AA). AA can be converted into prostaglandins (PGs), leukotrienes (LTs) and hydroxyeicosatetraenoic acids (HETEs) ${ }^{3}$ by cyclooxygenases (COXs), lipoxygenases (LOXs) and cytochrome P450 epoxygenases (CYP450). 5-LOX-derived $\mathrm{LTA}_{4}$ can be converted to lipoxins in the presence of 15-LOX, while 15-LOX generates 15-HETE ${ }^{3}$. Lipoxins and 15-HETE have been reported to exert anti-inflammatory activity; for example, LXA $_{4}$ and 15-HETE inhibit $\mathrm{LTB}_{4}$-induced chemotaxis of neutrophils ${ }^{4-6}$. Thromboxane $\mathrm{B}_{2}\left(\mathrm{TXB}_{2}\right)$ is non-enzymatically hydrolyzed from 
COX-derived $\mathrm{TXA}_{2}$, a potent bronchial smooth muscle spasmogen ${ }^{7}$, is known to reduce $\mathrm{T}$ cell secretion of the Th1 cytokine and favor T cell differentiation toward a Th2 cytokine profile ${ }^{8}$.

Together, these functionally diverse classes of eicosanoids are thought to play a critical role in maintaining homeostasis and have been an active area of investigation in assessing the mechanism underlying asthma and their potential utility in monitoring disease progression and treatment outcome. Indeed, several studies have suggested their roles as the biomarkers for screening, diagnosis, and, to a limited extent, monitoring the treatment outcome. For example, several independent studies have shown elevated levels of eicosanoids in the $\mathrm{EBC}$ of patients with asthma ${ }^{9,10}$, but due, perhaps in part, to the limited sample sizes and the heterogeneity of the study patient populations, unified evidence is currently lacking. As the result, knowledge about eicosanoids in disease progression and therapeutic outcome remains incomplete, and their relationship with the disease status has yet to be comprehensively explored and their clinical utility as biomarkers remains to be determined. We have previously reported that in a pediatric study population in Taiwan, the levels of exhaled $\mathrm{LTB}_{4}, \mathrm{LTE}_{4}, \mathrm{LXA}_{4}$, and $\mathrm{PGE}_{2}$ in asthmatic children were significantly different from those of healthy controls, and the combination of exhaled $\mathrm{LTB}_{4}$ and $\mathrm{LXA}_{4}$, together with FeNO and $\mathrm{FEV}_{1}$, best characterized childhood asthma ${ }^{11}$. We described herein an analysis of the levels of exhaled eicosanoids differed at the time of acute exacerbation and convalescence and reported that the levels of $\mathrm{TXB}_{2}$ and 15-HETE were the most responsive to therapy.

\section{Materials and Methods}

\section{Study subject}

A total of 393 bronchial asthmatic children aged between 5 and 12 years, consisting of 318 stable asthmatics and 75 acute asthma attack sufferers ( 47 males and 28 females), were recruited from the pediatric clinics of the Chang Gung Memorial Hospital, Taiwan, as a part of the ongoing PATCH study (Prediction of Allergies in Taiwanese Children). A total of 97 (59 males and 38 females) age-matched healthy subjects were enrolled from an elementary school in Taoyuan City, Taiwan. The diagnosis and classification of the clinical severity of asthma followed the published guidelines ${ }^{12}$. Asthma severity was categorized as mild (intermittent), moderate and severe, based on the previously described criteria ${ }^{11}$. An acute asthma attack was defined as a patient with dyspnea symptoms and audible expiratory wheeze accompanied by a $20 \%$ reduction in $\mathrm{FEV}_{1}$. Levels of EBC eicosanoids, $\mathrm{FEV}_{1}$, and $\mathrm{FeNO}$ were measured during acute asthma attack episodes and at two weeks after the acute asthma attacks. For the management of acute asthma exacerbation, all of the asthmatic children received terbutaline inhalation and oral prednisolone $1 \mathrm{mg} / \mathrm{kg} /$ day for 3 days. In the two weeks prior to EBC collection, none of the patients took medication containing antipyretics or anti-platelet agents. The healthy, non-asthmatic, and non-allergic subjects served as normal controls. This study was approved by the Humane Research Committee of the Chang Gung Memorial Hospital and informed consents were obtained from patients' parents or guardians prior to the start of the study. Additional phenotypes included BMI, serum IgE levels, $\mathrm{FEV}_{1}$, and FeNO measured as previously described ${ }^{13}$.

\section{Exhaled breath condensate (EBC) collection and exhaled nitric oxide measurement}

EBC was collected by Turbo-Deccs system (Medivac, Parma, Italy). Approximately $1 \mathrm{ml}$ of EBC was collected and immediately stored at -80 for further analysis. Following EBC collection (after 30 minutes of rest), fractional exhaled nitric oxide (FeNO) was measured according to published standards by using NIOX MINO Airway Inflammation Monitor (Aerocrine, Solna, Sweden).

\section{Lung function tests, methacholine challenge tests and analysis of eicosanoids}

The lung function tests were performed with the spirometer-Lungest 1000 (MES, Krakow, Poland) according to ERS/ATS ${ }^{14}$ standards. These children had not received any anti-asthmatic medication, including oral steroids, for at least two days.

Utilizing established solid phase extraction approach for the collection and purification of eicosanoids in EBCs, a panel of 6 eicosanoid species derived from arachidonic acids, representing products from major enzymatic pathways, was selected for initial discovery phase of the study population consisting of 60 asthmatics 
and 20 healthy controls, who were randomly selected from among the study populations. Eicosanoids were extracted from $\mathrm{EBCs}^{15}$ and measured with the respective enzyme immunoassay kit (Cayman Chemical, Ann Arbor, Michigan and Neogen, Lexington, Ky) as described previously ${ }^{11}$.

\section{Statistical analysis}

The significance of differences between the asthmatic and healthy children in their categorical variables was estimated by the $\chi^{2}$ test and continuous variables (for example, age, BMI, $\Delta \% \mathrm{FEV}_{1}$ ) by the $t$ test or ANOVA analyses. Receiver operating characteristic (ROC) curve with analysis of differences in the area under curves (AUC) was used to estimate the diagnostic accuracy. Furthermore, asthmatic subjects with repeated data were further divided into three groups according to the levels of eicosanoid species during exacerbation, i.e. the top 30\%, middle $40 \%$, and bottom 30\%. A two-way mixed-design analysis of variance (i.e. split-plot ANOVA) was performed for analyzing the effect of stratified eicosanoid levels (top $30 \%$ vs. middle $40 \%$ vs. bottom 30\%) and phases (active exacerbation vs. convalescence).

\section{Results}

\section{Analysis of exhaled eicosanoid species for differentiating asthma from normal controls}

In the discovery phase of the study, in addition to those previously reported ${ }^{11}$, including $\mathrm{LTB}_{4}$, the level of 15 HETE, but not $\mathrm{TXB}_{2}$, in EBCs of subjects with asthma $(\mathrm{N}=60)$ was significantly lower than that noted in the control group ( $\mathrm{N}=20)$ (Data not shown). To confirm the validity of these eicosanoid species in differentiating asthma patients from normal subjects, a total of 415 children were included in the validation phase, which consisted of 318 stable asthmatic and 97 healthy subjects. The demographics of these asthmatic children and healthy children are summarized in Table 1. Significant differences were noted for age, gender, serum total $\mathrm{IgE}, \mathrm{FEV}_{1}$ and FeNO between the subjects in the asthmatic and the control groups (all had $\mathrm{p}<0.001$ except for age and gender with $\mathrm{p}<0.05$ and $\mathrm{p}<0.01$, respectively; Table 1). No significant difference was found for BMI. In the expanded case-control design, the levels of exhaled 15-HETE were significantly lower for asthmatic subjects than for healthy subjects $\left(\mathrm{p}<0.0001\right.$; Table 2), while the level of $\mathrm{TXB}_{2}$ was similar between the two groups. Correlation analysis revealed that in asthmatic children, there was a significant positive correlation between the levels of $\mathrm{TXB}_{2}$ and those of $\mathrm{LTB}_{4}$ and $\mathrm{PGE}_{2}$ (Supplementary Figs. S1A and $\mathrm{S} 1 \mathrm{~B}$ ) in the exhaled condensate. Moreover, among the asthmatic subjects, negative correlations were found for $\mathrm{TXB}_{2}$ and $\mathrm{FEV}_{1}$, and also for 15-HETE and $\mathrm{LTB}_{4},(\mathrm{r}=-0.13, \mathrm{p}<0.05 ; \mathrm{r}=-0.11, \mathrm{p}<0.05$, respectively; Supplemental Figs. S1C and S1D).

When the asthmatic population was stratified into different severity groups (Table 2), it was noted that in comparison to the mild group, the moderate group was characterized with lower levels of exhaled 15HETE, and the severe group exhibited even lower levels. The difference in 15-HETE levels between healthy subjects and all three asthmatic severity groups was significant, but no significant difference was found between groups for $\mathrm{TXB}_{2}$. Further, as 15-HETE is known to exert inhibitory effect on 5-LOX-derived proinflammatory leukotrienes, the ratios of exhaled 15 -HETE/LTB 4 were calculated, and the results showed that the ratio of 15 -HETE/LTB 4 was significantly lower in subjects with severe asthma $(\mathrm{p}<0.01$; Table 2 ). We then utilized the data of Table 2 to generate the ROC curves and calculated the AUC values for each eicosanoid species. Figure 1 shows the ROC curves and the AUC values of the analyzed eicosanoids in differentiating asthma from healthy controls. Results showed a similar discriminating power for exhaled 15-HETE, FEV 1 and FeNO (Fig. 1).

\section{Assessment of the relationship between the levels of exhaled eicosanoids, $\mathrm{FEV}_{1}$, and FeNO with steroid responsiveness}

To assess whether the levels of exhaled eicosanoids varied during exacerbation and after convalescence, the levels of the exhaled eicosanoids, FeNO and $\mathrm{FEV}_{1}$ in asthmatic children (N=75; Supplementary Table S1) at acute exacerbation and convalescence stages were measured. As shown in Fig. 2, while the level of FeNO was not at variance between these two stages (Fig. 2A), there was a significant enhancement in the level of $\mathrm{FEV}_{1}$ (Fig. 2B; $\mathrm{p}<0.001$ ), and 15-HETE (Fig. $2 \mathrm{C}, \mathrm{p}<0.001$ ), and a significant reduction in the $\mathrm{TXB}_{2}$ (Fig. 
2D, $\mathrm{p}<0.05)$ level, while, as a group, the levels of $\mathrm{LTB}_{4}, \mathrm{LTE}_{4}, \mathrm{LXA}_{4}$ and $\mathrm{PGE}_{2}$ did not reveal significant difference (Supplemental Fig. S2) before and after prednisone treatment. Furthermore, when the respective levels of each eicosanoid species were stratified into those at the 30th percentile, significant changes were particularly noted for those with higher initial levels of both 15-HETE and $\mathrm{TXB}_{2}$ at the exacerbation phase (Fig. 3); also, significant decreases for exhaled $\mathrm{LTB}_{4}, \mathrm{LTE}_{4}, \mathrm{LXA}_{4}$, and $\mathrm{PGE}_{2}$ were noted (all with $\mathrm{p}<0.001$; Supplemental Fig. S3) for those at the top 30 percentile.

\section{Discussion}

In a study population consisting of 318 children with asthma, lower levels of 15-HETE were found. ROC analysis of individual parameters demonstrated similar levels of the sensitivity and specificity between exhaled 15-HETE and two commonly used parameters in monitoring asthma, $\mathrm{FEV}_{1}$ and $\mathrm{FeNO}^{16}$. Further, positive correlations were found between the levels of $\mathrm{TXB}_{2}$ and those of $\mathrm{LTB}_{4}$ and $\mathrm{PGE}_{2}$ in asthmatic children. Also, among the asthmatic subjects, negative correlations were found for $\mathrm{TXB}_{2}$ and $\mathrm{FEV}_{1}$, and for 15-HETE and $\mathrm{LTB}_{4}$. Among those parameters analyzed, reduced levels of $\mathrm{TXB}_{2}$, but increased levels of 15-HETE, were noted after 3 days of oral prednisolone treatment, concomitant with the improvement of lung function in asthmatic children. When the asthmatic population was stratified into different severity groups, it was noted that the ratio of $15-\mathrm{HETE} / \mathrm{LTB}_{4}$ was significantly lower in subjects with severe asthma. Furthermore, when we investigated changes in the levels of 15-HETE and $\mathrm{TXB}_{2}$ during exacerbation and convalescence in subjects according to the top 30\%, middle $40 \%$, and bottom $30 \%$ (as determined at the exacerbation levels), it was found that higher initial exacerbation would have responded well to prednisone treatment. These results, collectively, suggest their potential utility as a new set of lipid markers for monitoring asthma and its therapeutic outcome.

The family of eicosanoids is the most prevalent lipid mediators providing both pro-inflammatory signals and terminating the inflammatory process. Eicosanoid profiling in the exhaled breath condensate is complementary to the cellular phenotyping of asthmatic inflammation ${ }^{17}$. Our findings revealed that the levels of 15-HETE were significantly reduced in the EBCs of asthmatic subjects as compared to that of healthy controls, but was increased after treatment. Kowal et al also reported that 15-HETE in asthma patients was significantly lower than in healthy subjects ${ }^{15}$. Song et al demonstrated that 15-HETE regulated MUC5AC expression via modulating MMP-9, MEK/ERK/Sp-1, and PPAR $/$ PTEN/ Akt signaling pathways in PMAtreated respiratory epithelial cells ${ }^{18}$. Also, high 12/15-LOX activity and 15-HETE levels have been suggested to be indicative of pro-inflammatory responses in asthma ${ }^{19,20}$. Besides the anti-inflammatory effects, 15HETE has been shown to be an endogenous ligand for PPAR $\gamma$ (peroxisome proliferator-activated receptor gamma), which has anti-inflammatory effects such as regulating inflammatory cytokines ${ }^{21,22}$, neutrophil migration and mucin secretion ${ }^{18}$. For instance, the PPAR $\gamma$ agonist rosiglitazone has been shown to display bronchodilator effects in a group of patients with glucocorticoids-resistant asthma ${ }^{23}$. The reduction of 15-HETE may, therefore, suggest its close relationship with asthma and warrant further investigation.

Moreover, as 15-HETE may exert their anti-inflammatory effect through inhibiting 5-LOX-derived proinflammatory leukotrienes ${ }^{24}$, we also calculated the ratio of exhaled 15-HETE:LTB ${ }_{4}$ and found significantly lower in subjects with severe asthma. The mean 15-HETE:LTB ${ }_{4}$ ratio was $79 \%$ lower in patients with severe asthma when compared with that in patients with moderate asthma $(\mathrm{P}<0.01)$. These findings suggest that 15-HETE biosynthetic capacity might be defective in patients with severe asthma and thus contribute to the perpetuation of airway inflammation in these patients. Moreover, $\mathrm{TXA}_{2}$ is a lipid mediator and a bronchoconstrictor contributing to the pathophysiology of asthma ${ }^{7}$, while $\mathrm{TXB}_{2}$ is a stable metabolite of $\mathrm{TXA}_{2}$. The reduction of $\mathrm{TXB}_{2}$ levels might be indicative of steroid's effect and a marker responsive to the intervention.

While, consistent with a previous report ${ }^{25}$, but not the others ${ }^{13,26,27}$, we did not find difference in the level of exhaled $\mathrm{TXB}_{2}$ (and its metabolite, 11-dihydro- $\mathrm{TXB}_{2}$; data not shown) between asthmatic and healthy children, but the level of $\mathrm{TXB}_{2}$ showed significant reduction after 3 days of oral prednisolone treatment. Further, Dworski et al. found that prednisone was able to reduce the synthesis of eicosanoids, including $\mathrm{TXB}_{2}$ level, in macrophage-rich BAL-fluid cells from 14 atopic asthmatic volunteers at baseline and after 
allergen instillation $^{28}$. It is also worth noting that in double-blind, placebo-controlled trials, the thromboxane receptor antagonist, seratrodast, and the thromboxane synthase inhibitor, ozagrel, were proven efficacious in the treatment of patients with asthma ${ }^{29}$. However, the effect of $\mathrm{TXA}_{2}$ inhibitors in asthma has not been widely used because no statistically significant difference was observed, but it has been suggested that it might be a good disease marker of asthma only in a certain ethnic group ${ }^{30}$. One explanation for these conflicting results could be phenotypically different in the study population. Nevertheless, while the level of $\mathrm{TXB}_{2}$ may be dependent on the stage of asthma and its severity, the reduction in $\mathrm{TXB}_{2}$ after therapy appears to be consistent. Further independent studies are needed to confirm these results. The finding that the level of exhaled $\mathrm{TXB}_{2}$ was significantly reduced during convalescence is significant in and of itself, providing a basis for further exploring its clinical utility in monitoring the therapeutic outcome in place of FeNO.

Furthermore, it is worth noting that $\mathrm{LTB}_{4}, \mathrm{LTE}_{4}, \mathrm{PGE}_{2}$ and $\mathrm{LXA}_{4}$ also showed reduction in patients with the respective levels at the $30 \%$ percentile, and, in fact, only in those who had higher levels of exhaled eicosanoids. This could be related to the stages of asthma progression during exacerbation, and to the phenotypic heterogeneity of asthma in the study population in terms of its etiology and pathogenic mechanism. Further investigation into this possibility is clearly required. In conclusion, these results provided insight into the measurements of exhaled eicosanoid profiles, and showed that there was a significant difference between the levels of $\mathrm{TXB}_{2}$ and 15-HETE during acute asthma exacerbation and convalescence. Additional prospective studies are necessary to evaluate the utility of the proposed discriminator diagnosis and monitoring of childhood asthma.

Acknowledgments: This work was supported by Chang Gung Memorial Hospital (CMRPG3G2051, CMRPVVK0161)., the Ministry of Science and Technology, Taiwan (MOST 108-2314-B-182A-088) and, in part, by a grant from National Health Research Institutes, Taiwan (EO-109-PP-10).

Impact statement: The exhaled 15-HETE was found to discriminate childhood asthma while decreased levels of exhaled $\mathrm{TXB}_{2}$ and increased levels of 15-HETE were prominent after treatment.

Conflict of interest : The authors declare that they have no conflict of interests

\section{References}

1. Anderson GP. Endotyping asthma: new insights into key pathogenic mechanisms in a complex, heterogeneous disease. The Lancet, vol. 372, no. 9643, pp. 1107-1119, 2008.

2. Harizi H, Corcuff JB, Gualde N. Arachidonic-acid-derived eicosanoids: roles in biology and immunopathology. Trends Mol Med. 2008;14:461-9.

3. Dennis EA, Norris PC. Eicosanoid storm in infection and inflammation Nat Rev Immunol. 2015; 15: $511-523$.

4. Merched AJ, Ko K, Gotlinger KH, Serhan CN, Chan L: Atherosclerosis: evidence for impairment of resolution of vascular inflammation governed by specific lipid mediators. FASEB J 2008, 22:3595-3606.

5. Takata S, Matsubara M, Allen PG, Janmey PA, Serhan CN, Brady HR: Remodeling of neutrophil phospholipids with 15(S)-hydroxyeicosatetraenoic acid inhibits leukotriene B4-induced neutrophil migration across endothelium. J Clin Invest 1994, 93:499-508.

6. Serhan CN, Chiang N, Van Dyke TE. Resolving inflammation: dual anti-inflammatory and pro-resolution lipid mediators. Nat Rev Immunol. 2008;8:349-61.

7. Dogné JM, de Leval X, Benoit P, Rolin S, Pirotte B, Masereel B. Therapeutic potential of thromboxane inhibitors in asthma. Expert Opin Investig Drugs. 2002;11:275-81.

8. Li H, Edin ML, Gruzdev A, Cheng J, Bradbury JA, Graves JP, DeGraff LM, Zeldin DC. Regulation of T helper cell subsets by cyclooxygenases and their metabolites. Prostaglandins Other Lipid Mediat. 2013;104105:74-83. 
9. Mastalerz L, Sanak M, Kumik J, Gawlewicz-Mroczka A, Celejewska-W'ojcik N, C’miel A, Szczeklik A. Exhaled eicosanoids following bronchial aspirin challenge in asthma patients with and without aspirin hypersensitivity: The pilot study. J Allergy, 2012, Article ID 696792.

10. Carraro S, Corradi M, Zanconato S, Alinovi R, Pasquale MF, Zacchello F, Baraldi E. Exhaled breath condensate cysteinyl leukotrienes are increased in children with exercise-induced, bronchoconstriction. J Allergy Clin Immunol 2005;115:764-770.

11. Chen LC, Tseng HM, Kuo ML, Chiu CY, Liao SL, Su KW, Tsai MH, Hua MC, Lai SH, Yao TC, Yeh KW, Wu AH, Huang JL, Huang SK. A composite of exhaled LTB4, LXA4, FeNO and FEV1 as an "asthma classification ratio" characterizes childhood asthma. Allergy. 2018;73:627-634.

12. Bacharier LB, Boner A, Carlsen KH, Eigenmann PA, Frischer T, Gotz M, Helms PJ, Hunt J, Liu A, Papadopoulos N, Platts-Mills T, Pohunek P, Simons FE, Valovirta E, Wahn U, Wildhaber J. European Pediatric Asthma Group: Diagnosis and treatment of asthma in childhood: a PRACTALL consensus report. Allergy 2008;63:5-34.

13. Joint Statement of the American Thoracic Society (ATS) and the European Respiratory Society (ERS). ATS/ERS recommendations for standardized procedures for the online and offline measurement of exhaled lower respiratory nitric oxide and nasal nitric oxide, 2005. Am J Respir Crit Care Med 2005;171:912-930.

14. Miller MR, Hankinson J, Brusasco V, Burgos F, Casaburi R, Coates A, Crapo R, Enright P, van der Grinten CP, Gustafsson P, Jensen R, Johnson DC, MacIntyre N, McKay R, Navajas D, Pedersen OF, Pellegrino R, Viegi G, Wanger J. Standardisation of spirometry. Eur Respir J 2005;26:319-338.

15. Kowal1 K, Zukowski1 S, Kowal-Bielecka O, Bodzenta-Lukaszyk A, DuBuske LM. Concentrations of 15-HETE and PGE2 in Exhaled Breath Condensates of Asthma Patients J ALLERGY CLIN IMMUNOL FEBRUARY 2008.

16. Venkatnarayan Kavitha, Anant Mohan, Karan Madan, Vijay Hadda, GC Khilnani, and Randeep Guleria Fractional exhaled nitric oxide is a useful adjunctive modality for monitoring bronchial asthma Lung India. 2017; 34(2): 132-137.

17. Marek Sanak Eicosanoid Mediators in the Airway Inflammation of Asthmatic Patients: What is New? Allergy Asthma Immunol Res. 2016; 8(6): 481-490.

18. Song YS, Kim MS, Lee DH, Oh DK, Yoon DY. 15-Hydroxyeicosatetraenoic Acid Inhibits Phorbol-12Myristate-13- Acetate-Induced MUC5AC Expression in NCI-H292 Respiratory Epithelial Cells J. Microbiol. Biotechnol. 2015;25:589-597.

19. Liu, C.; Xu, D.; Liu, L.; Schain, F.; Brunnstrom, A.; Bjorkholm, M.; Claesson, H.E.; Sjoberg, J. 15lipoxygenase-1 induces expression and release of chemokines in cultured human lung epithelial cells. Am. J. Physiol. Lung Cell Mol. Physiol., 2009, 297(1), L196-203.

20. Chu H, Balzar S, Westcott JY, Trudeau JB, Sun Y, Conrad DJ, Wenzel SE. Expression and activation of 15- lipoxygenase pathway in severe asthma: relationship to eosinophilic phenotype and collagen deposition. Clin. Exp. Allergy, 2002, 32(11), 1558-1565.

21. Mabalirajan U, Agrawal A, Ghosh B. 2012. 15-Lipoxygenase eicosanoids are the putative ligands for vanilloid receptors and peroxisome proliferator-activated receptors (PPARs). Proc. Natl. Acad. Sci. USA 109: E1; author reply E2

22. Chen GG, Xu H, Lee JF, et al. 15-hydroxy-eicosatetraenoic acid arrests growth of colorectal cancer cells via a peroxisome proliferator-activated receptor gamma-dependent pathway. Int J Cancer 2003;107:837e43.

23. Spears M, Donnelly I, Jolly L, Brannigan M, Ito K, McSharry C, Lafferty J, Chaudhuri R, Braganza G, Bareille P, Sweeney L, Adcock IM, Barnes PJ, Wood S, Thomson NC. Bronchodilatory effect of the PPAR-gamma agonist rosiglitazone in smokers with asthma. Clin. Pharmacol. Ther., 2009, 86(1), 49-53. 
24. Takata S, Matsubara M, Allen PG, Janmey PA, Serhan CN, Brady HR: Remodeling of neutrophil phospholipids with 15(S)-hydroxyeicosatetraenoic acid inhibits leukotriene B4-induced neutrophil migration across endothelium. J Clin Invest 1994, 93:499-508.

25. Mondino C, Ciabattoni G, Koch P et al. Effects of inhaled corticosteroids on exhaled leukotrienes and prostanoids in asthmatic children. J Allergy Clin Immunol 2004; 114:761-7.

26. Huszar E, Szabo Z, Jakab A, Barta I, Herjavecz I, Horvath I. Comparative measurement of thromboxane A2 metabolites in exhaled breath condensate by different immunoassays. Inflamm Res 2005; 54:350-5.

27. Ma N, Shang W, Qin J. The clinical significance of measurement of TXB2 and 6-K-PGF1 $\alpha$ of plasma and bronchoalveolar lavage fluid in patients with bronchial asthma and chronic bronchitis. Labeled Immunoassays and Clinical Medicine 2002; 9;202-204.

28. Dworski R, Fitzgerald GA, Oates JA, Sheller JR. Effect of oral prednisone on airway inflammatory mediators in atopic asthma. Am J Respir Crit Care Med. 1994;149:953-9.

29. Dogné JM, de Leval X, Benoit P, Delarge J, Masereel B. Thromboxane A2 inhibition: therapeutic potential in bronchial asthma. Am J Respir Med. 2002;1(1):11-7.

30. Takaku Y, Kurashima K, Kobayashi T, Nakagome K, Nagata M. Eicosanoids in exhaled breath condensate of airway inflammation in patients with asthma. Allergol Int. 2016 pii: S1323-8930;30062-4.

Table 1. Demographics of study subjects.

\begin{tabular}{llll}
\hline Parameter & Asthmatic(N=318) & Healthy $(\mathrm{N}=97)$ & $\mathrm{P}$ value \\
\hline Age & $8.58 \pm 0.16$ & $8.93 \pm 0.2$ & $\mathrm{P}<0.05$ \\
Gender (boy/girl) & $205 / 113$ & $59 / 38$ & $\mathrm{P}<0.01$ \\
BMI category, N (\%) & & & \\
Underweight & $11(3.5 \%)$ & $7(7.2 \%)$ & \\
Normal & $203(63.8 \%)$ & $56(57.7 \%)$ & \\
Overweight & $52(16.4 \%)$ & $20(20.6 \%)$ & \\
Obesity & $52(16.4 \%)$ & $14(14.4 \%)$ & \\
Asthma severity, N (\%) & & & \\
Mild & $227(71.4 \%)$ & $\mathrm{ND}$ & \\
Moderate & $62(19.5 \%)$ & $\mathrm{ND}$ & \\
Severe & $29(9.1 \%)$ & $\mathrm{ND}$ & \\
Serum total IgE & $561.1 \pm 39.5$ & $68.2 \pm 5.1$ & $\mathrm{P}<0.001$ \\
FEV 1\% & $72.3 \pm 1.0$ & $85.2 \pm 1.2$ & $\mathrm{P}<0.001$ \\
FeNO,ppb & $23.5 \pm 1.2$ & $10.3 \pm 0.7$ & $\mathrm{P}<0.001$ \\
PC 20 & $8.6 \pm 0.6$ & $\mathrm{ND}$ & \\
ECP(ng/L) & $30.4 \pm 10.9$ & $\mathrm{ND}$ & \\
\hline
\end{tabular}

Table 2. Levels of 15-HETE and $\mathrm{TXB}_{2}$ in subjects in the validation phase and asthmatic subjects stratified by severity.

\begin{tabular}{|c|c|c|c|c|c|c|c|}
\hline & $\mathrm{N}$ & $\begin{array}{l}15-\mathrm{HETE}^{\mathrm{a}} \\
\text { mean } \pm \text { S.E. }\end{array}$ & $\mathrm{P}$ value & $\begin{array}{l}15- \\
\text { HETE/LTB }{ }_{4}^{a} \\
\text { ratio }\end{array}$ & $P$ value & $\begin{array}{l}\mathrm{TXB}_{2} \\
\text { mean } \pm \text { S.E. }\end{array}$ & $\mathrm{P}$ value \\
\hline Healthy & 97 & $\begin{array}{l}209.7 \pm \\
22.93\end{array}$ & & $\begin{array}{l}144.63 \pm \\
17.93\end{array}$ & & $\begin{array}{l}8.13 \pm \\
1.92\end{array}$ & \\
\hline Asthma $^{b}$ & 318 & $\begin{array}{l}72.71 \pm \\
7.67\end{array}$ & $<0.0001$ & $\begin{array}{l}35.17 \pm \\
4.13\end{array}$ & $<0.01$ & $\begin{array}{l}8.16 \pm \\
1.06\end{array}$ & $\mathrm{NS}$ \\
\hline
\end{tabular}




\begin{tabular}{|c|c|c|c|c|c|c|c|}
\hline & $\mathrm{N}$ & $\begin{array}{l}15-\text { HETE }^{\mathrm{a}} \\
\text { mean } \pm \text { S.E. }\end{array}$ & $\mathrm{P}$ value & $\begin{array}{l}15- \\
\text { HETE } / \mathrm{LTB}_{4}{ }^{\mathrm{a}} \\
\text { ratio }\end{array}$ & $\mathrm{P}$ value & $\begin{array}{l}\mathrm{TXB}_{2} \\
\text { mean } \pm \text { S.E. }\end{array}$ & $\mathrm{P}$ value \\
\hline Mild & 227 & $\begin{array}{l}76.19 \pm \\
9.03\end{array}$ & $<0.0001$ & $\begin{array}{l}38.92 \pm \\
5.04\end{array}$ & $<0.01$ & $\begin{array}{l}8.21 \pm \\
1.25\end{array}$ & NS \\
\hline Moderate & 62 & $\begin{array}{l}71.02 \pm \\
17.4\end{array}$ & $<0.0001$ & $\begin{array}{l}34.88 \pm \\
7.06\end{array}$ & $<0.01$ & $8.60 \pm 2.4$ & NS \\
\hline Severe & 29 & $\begin{array}{l}49.50 \pm \\
25.44\end{array}$ & $<0.0001$ & $\begin{array}{l}6.92 \pm \\
2.58\end{array}$ & $<0.01$ & $6.81 \pm 3.5$ & NS \\
\hline
\end{tabular}

${ }^{a}$ Asthma group or each of its severity groups versus healthy control group.

b Among the subgroups of asthma, there are no significant differences from each other.

NS, not significant.

Figure legends

Table 1. Demographics of study subjects.

Table 2. Levels of 15-HETE and $\mathrm{TXB}_{2}$ in subjects in the validation phase and asthmatic subjects stratified by severity

Figure 1. ROC analysis of exhaled 15-HETE, $\mathrm{TXB}_{2}, \mathrm{FeNO}$ and $\Delta \% \mathrm{FEV} 1$.

Figure 2. Changes in the levels of FeNO, $\mathrm{FEV}_{1}$ and eicosanoid species during exacerbation and convalescence. The levels of (A) FeNO, (B) $\mathrm{FEV}_{1}$, (C) 15-HETE and (D) $\mathrm{TXB}_{2}$ during acute exacerbation (AE) and after treatment (C, convalescence) in a total of 75 children with asthma. Each line represents each individual sample. Note: All p-values were adjusted for multiple testing by Holm methods.

Figure 3. Changes in the levels of $\mathrm{TXB}_{2}$ and 15-HETE during exacerbation and convalescence in subjects according to the top $30 \%$, middle $40 \%$, and bottom $30 \%$ (as determined at the exacerbation levels). FA denotes the between-subjects main effect of stratified eicosanoid levels during exacerbation; FB denotes the within-subjects main effect of phasic change; FAXB denotes the interaction of FA and FB variables.

\section{Supplemental Figure legends}

Supplemental Table S1. Demographic comparison of acute asthmatics.

Supplemental Figure S1. Correlation analysis of $\mathrm{TXB}_{2}$ with (A) $\mathrm{FEV}_{1}$, (B) $\mathrm{LTB}_{4}$, (C) $\mathrm{PGE}_{2}$ and of (D) 15-HETE and $\mathrm{LTB}_{4}$.

Supplemental Figure S2. Levels of (A) $\mathrm{LTB}_{4}$, (B) $\mathrm{LTE}_{4}$, (C) Lipoxin $\mathrm{A}_{4}$, and (D) $\mathrm{PGE}_{2}$ in EBCs of asthmatic children at acute exacerbation and convalescence stages.

Supplemental Figure S3. Levels of $\mathrm{LTB}_{4}(\mathrm{~A}), \mathrm{LTE}_{4}(\mathrm{~B})$, Lipoxin $\mathrm{A}_{4}(\mathrm{C})$, and $\mathrm{PGE}_{2}$ (D) during acute exacerbation (AE) and two weeks after oral prednisolone treatment $(\mathrm{C}$, convalescence) in asthmatic subjects with the respective eicosanoid levels at the 30th percentile at exacerbation. Each line represents each individual sample. Note: All p-values are adjusted for multiple testing by Holm methods. 

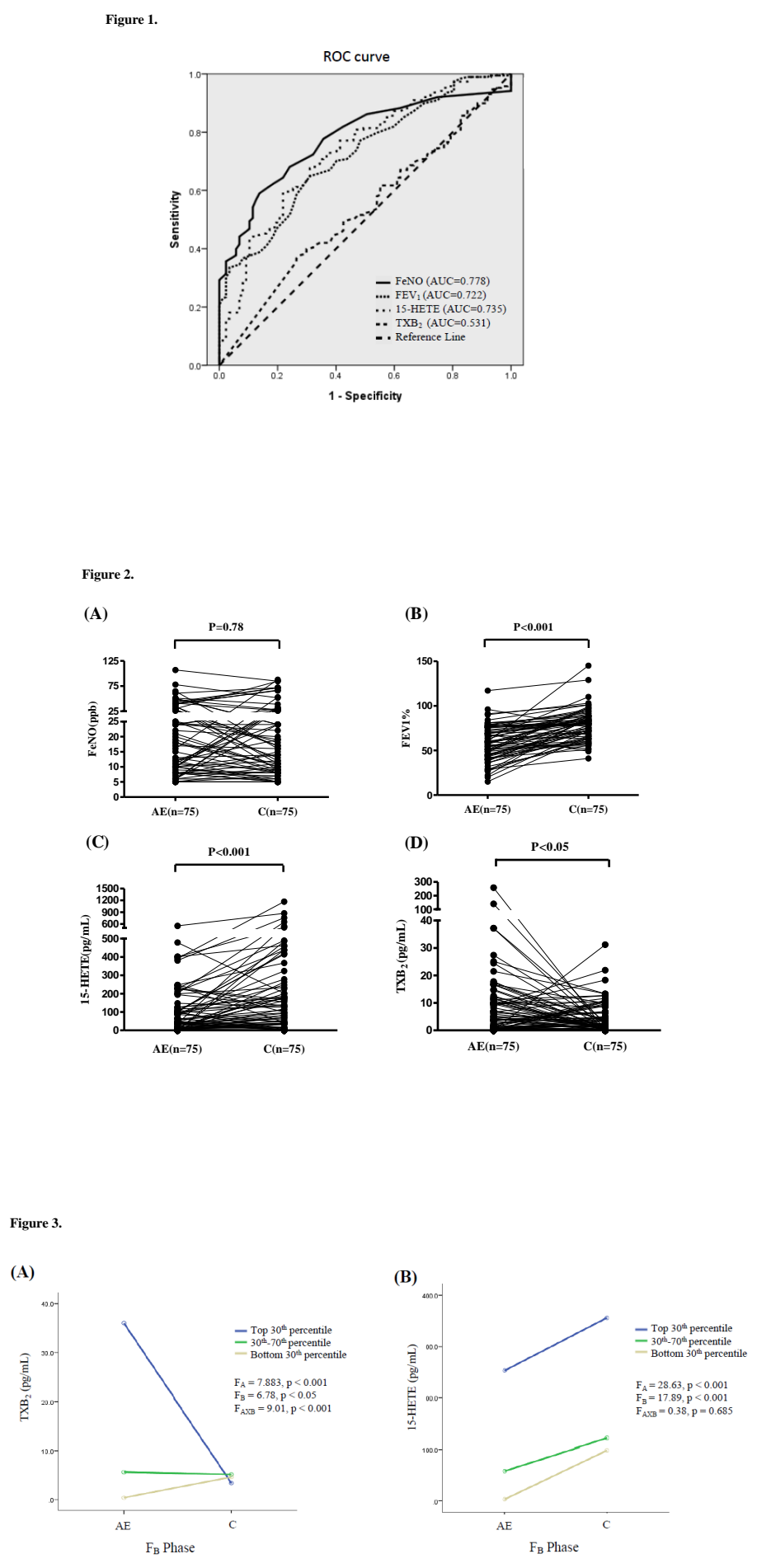